\title{
Analysis of Asymmetric Cantor Set Multi-Layered Structure
}

\author{
ABDUL KHALEQUE* \\ School of Engineering and Information Technology, The University of New South Wales, \\ Canberra, ACT 2610 Australia
}

(Received June 21, 2014; in final form November 3, 2014)

\begin{abstract}
This article studies the propagation of light in asymmetric Cantor set fractal multi-layered structures. We show that well designed Cantor set structures can lead to broadband high reflectivity over a large wavelength range but, by adding asymmetries to the Cantor set, it is possible to control the reflectivity from $100 \%$ to nearly $0 \%$.

DOI: $10.12693 /$ APhysPolA.126.1258

PACS: 42.79.Ci, 42.25.Bs
\end{abstract}

\section{Introduction}

The word fractal is derived from the latin word "fractus", meaning broken. In more scientific terms, fractal is used to describe geometries that present selfsimilar patterns, such as the small patterns are replicas of the large ones [1]. Fractal theory is applied to a wide range of areas including chemistry, biology, physics and engineering. In electromagnetics, fractals have been used to produce antennas [2], optical devices [3-5] and nanoparticles [6].

In particular, one-dimensional structures such as the Cantor set can be easily constructed and has been used to create broadband devices and enhancing nonlinear effects. The Cantor set has a Hausdorff dimension of log (2) $/ \log (3)$ [1]. A standard Cantor set is created by initially removing the middle third of a segment, creating a Cantor set of order 1 . The next order is obtained by removing the middle third of the first and third remaining segments - the procedure could be, in principle, repeated at exhaustion.

On the other hand, photonic crystals have been used in a wide variety of applications in filtering, sensors, lasers and multiplexing [7-17]. Photonic crystals are periodic structures of high refractive index contrast (e.g. GaAs and air): in a certain wavelength range (photonic bandgap region), light cannot propagate through the structure. In addition to producing reflectors that are used in lasers [10] and optical filters, defects introduced in the photonic crystal lattice can be used to create microcavities and waveguides. In fact, photonic crystal waveguides can exhibit very interesting properties such as very low group velocities.

In this paper, we analyze one-dimensional photonic crystal structures that are based on the Cantor set structures: we modify the traditional Cantor set structure by adding asymmetries in the formation of the Cantor set and study the reflectivity properties of these photonic

\footnotetext{
*e-mail: abdul.khaleque@student.adfa.edu.au
}

crystal structures. Adding asymmetries to the original photonic crystal structure can considerably tailor the reflectivity properties of the photonic crystal and, eventually, lead to a high electric fields. A MATLAB code is developed to simulate all devices in this paper based on the transfer matrix method [18].

\section{General description of the structure and theory}

The structure to be analyzed is shown in Fig. 1: order 0 structure consists of pure GaAs, order 1 structure consists of a feeding and collecting GaAs long plates with a Cantor structure with thickness for each layer of $l_{1}=s d_{1}$, $l_{2}=d_{1}$ and $l_{3}=s d_{1}$ where $d_{1}$ and $s$ are the optical thickness and distortion parameter of the structure, respectively, i.e., $d_{1}=d_{\text {ref }} / n_{\text {mat }}$ ( $d_{\text {ref }}$ is the reference physical thickness $=\lambda_{0} / 4$, where $\lambda_{0}$ is chosen to be $980 \mathrm{~nm}$ and $n_{\text {mat }}$ is the refractive index of the material: 1.0 for air and 3.521 for GaAs). Similarly, the thickness of each layer for order 2 structure are $l_{1}=s^{2} d_{1} / 3, l_{2}=s d_{1} / 3$, $l_{3}=s^{2} d_{1} / 3, l_{4}=d_{1}, l_{5}=s^{2} d_{1} / 3, l_{6}=s d_{1} / 3$ and $l_{7}=s^{2} d_{1} / 3$ (as shown in Fig. 1c).

\section{(a)}

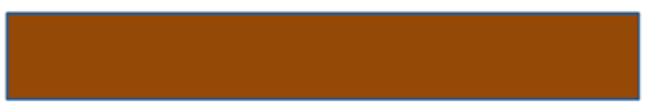

GaAs

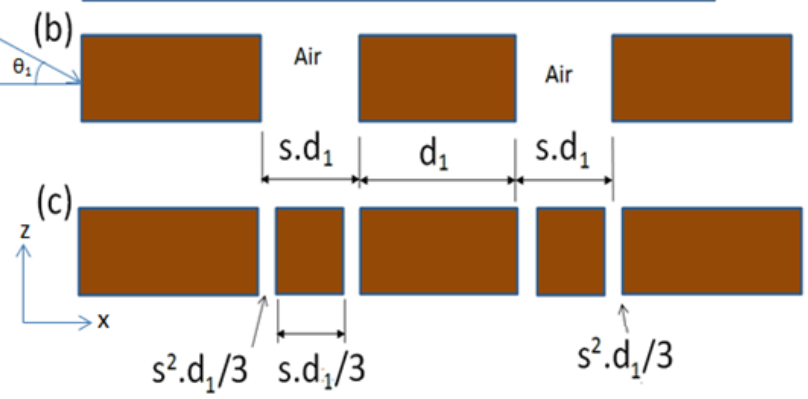

Fig. 1. Schematic of asymmetric Cantor set multilayered structure.

It is assumed that a plane wave with inclination angle $\left(\theta_{1}\right)$ is incident in the $x-z$ plane. Two types of modes, transverse electric (TE) and transverse magnetic (TM), 
can propagate in this structure. The electric field is considered in the $y$-direction and $x-z$ plane for TE mode and TM mode, respectively. For the $i$-th layer, the electric field can be expressed as [18]:

$$
E_{i}=A_{i} \exp \left(-\mathrm{j} k_{i x}\left(x-x_{i}\right)\right)+B_{i} \exp \left(\mathrm{j} k_{i x}\left(x-x_{i}\right)\right),(1)
$$
where $A_{i}$ and $B_{i}$ are incident and reflected field amplitude, $x_{i}=\sum_{k=2}^{i-1} l_{k}$ and $k_{i x}=(2 \pi / \lambda) n_{i} \cos \theta_{i}$ ( $\lambda$ is the free space wavelength and $\theta_{i}$ is the angle between the direction of propagation of the plane wave in the $i$-th layer and the $x$-axis).

In this structure, the input and output waves relationship are given by [18]:

$$
\left(\begin{array}{l}
A_{1} \\
B_{1}
\end{array}\right)=\left(\begin{array}{ll}
M_{11} & M_{12} \\
M_{21} & M_{22}
\end{array}\right)\left(\begin{array}{l}
A_{N} \\
B_{N}
\end{array}\right)
$$

where $M=D_{1}^{-1}\left[\Pi_{l=2}^{N-1} D_{l} P_{l} D_{l}^{-1}\right] D_{N}$. In case of $l$-th layer, $D_{l}$ matrix elements can be expressed as

$$
\begin{aligned}
& D_{l}=\left(\begin{array}{cc}
1 & 1 \\
n_{l} \cos \theta_{l} & -n_{l} \cos \theta_{l}
\end{array}\right), \quad \text { for TE waves } \\
& D_{l}=\left(\begin{array}{cc}
\cos \theta_{l} & \cos \theta_{l} \\
n_{l} & -n_{l}
\end{array}\right), \quad \text { for TM waves }
\end{aligned}
$$

For the $l$-th layer, the propagation matrix $P_{l}$ is given by [18]:

$$
P_{l}=\left(\begin{array}{cc}
\exp \left(\mathrm{j} \varphi_{l}\right) & 0 \\
0 & \exp \left(-\mathrm{j} \varphi_{l}\right)
\end{array}\right)
$$

with $\varphi_{l}=k_{l x} l_{l}$.

As it is possible to determine matrix $M$ according to the procedure previously described, the reflectivity $R$ can be expressed as [19]:

$$
R=\left|\frac{M_{21}}{M_{11}}\right|^{2} \text {. }
$$

\section{Results and discussions}

Figure 2a shows the reflectivity spectrum for the 1st order Cantor set structure of GaAs and air with $\theta_{1}=0^{\circ}$ (normal incidence) under $s=1.0$ (solid black curve), $s=0.8$ (dashed blue curve) and $s=0.6$ (solid green curve) for TE modes. In this case, the results for TE and TM modes are degenerate. No clear transmission window appears for $s=1.0$ (black curve), nonetheless high transmissivity appears between $1088 \mathrm{~nm}$ to $1186 \mathrm{~nm}$ and $855 \mathrm{~nm}$ to $943 \mathrm{~nm}$, for $s=0.8$ (blue dashed curve) and $s=0.6$ (green curve), respectively. There are two small peaks at $695 \mathrm{~nm}$ and $775 \mathrm{~nm}$ with reflectivity of 0.53 for $s=1.0$ (black curve), while only one peak (at $750 \mathrm{~nm}$ ) for $s=0.6$ (green curve). In addition to that one dip (around $747 \mathrm{~nm}$ ) and one bandgap (with reflectivity close to 1.0 ) were observed from nearly $750 \mathrm{~nm}$ to $1083 \mathrm{~nm}$ with asymmetry $(s=0.8)$. On the other hand, one bandgap (with reflectivity close to 1.0) is observed under $s=1.0$ (black curve) between $818 \mathrm{~nm}$ to $1223 \mathrm{~nm}$.

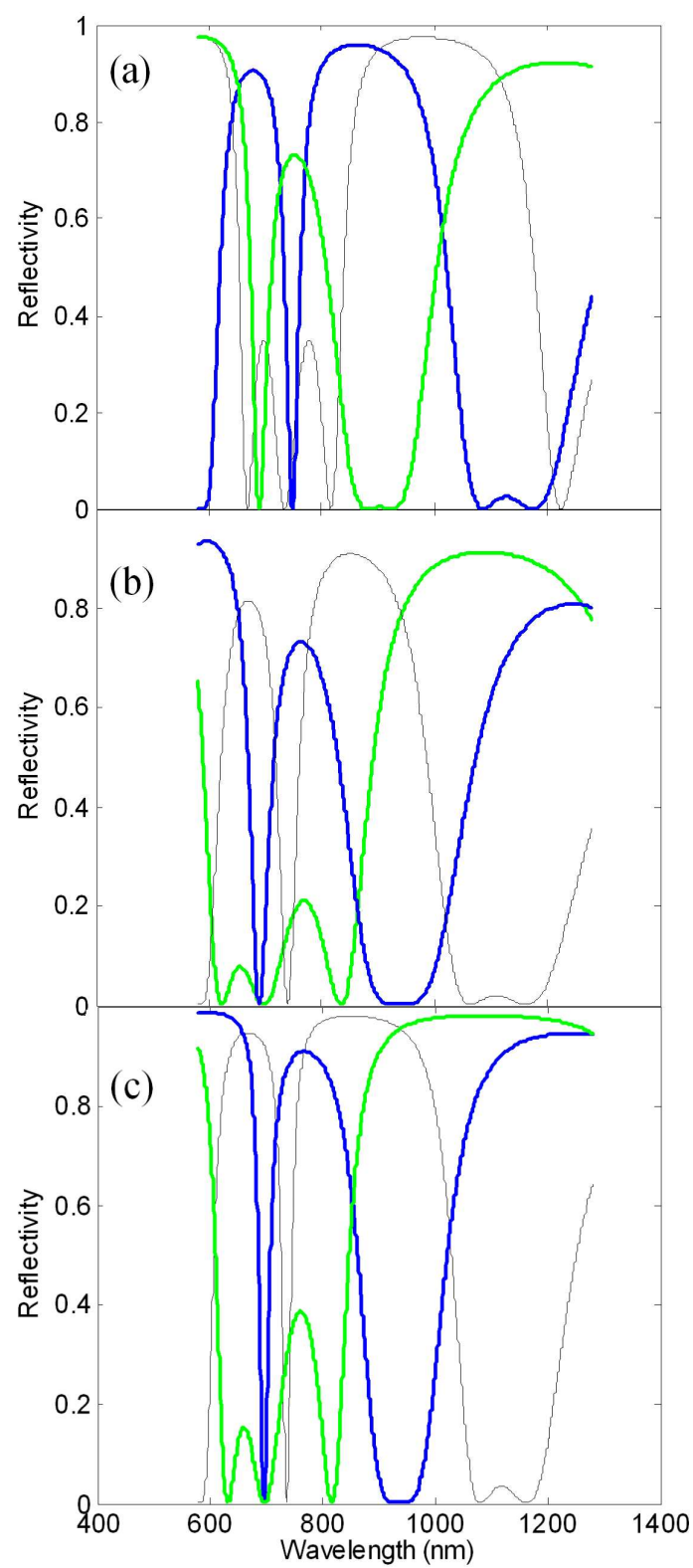

Fig. 2. Reflectivity spectrum of asymmetric Cantor set first-order GaAs-air structure for $s=1.0$ (solid thin black curve), 0.8 (dashed blue curve), 0.6 (solid thick green curve) for (a) TE mode with $\theta_{1}=0^{\circ}$, (b) TE mode with $\theta_{1}=10^{\circ}$, (c) TM mode with $\theta_{1}=10^{\circ}$.

Figure $2 \mathrm{~b}$ shows the reflectivity spectrum for TE modes with incidence angle of $10^{\circ}$. The bandgap (with reflectivity close to 1) occurs from approximately $740 \mathrm{~nm}$ to $1060 \mathrm{~nm}$ and $840 \mathrm{~nm}$ to $1270 \mathrm{~nm}$, respectively, for $s=1.0$ (black curve) and $s=0.6$ (green curve). In the case of $s=0.8$ (dashed blue curve), two transmission dips are observed around $700 \mathrm{~nm}$ and $950 \mathrm{~nm}$. TM mode with $\theta_{1}=10^{\circ}$ shows identical results (shown in Fig. 2c) as for TE mode with $\theta_{1}=10^{\circ}$ (as shown in Fig. 2b) but the amount of reflection is slightly greater for $s=1.0$ (black curve) and $s=0.8$ (blue curve). Therefore, the bandgap shifted to shorter wavelengths and is narrower. 
Figure 3 shows the field profiles as a function of distance $(\mu \mathrm{m})$ for the first order Cantor set structure under normal inclination $\left(\theta_{1}=0^{\circ}\right)$. In this case, TE waves and both types of structure, symmetrical and asymmetrical, are considered. The highest normalized electric field value is approximately 4.5 (as shown in Fig. 3b) with asymmetries $(s=0.6)$ in the original photonic crystal structures which is more than twice higher than the value obtained from photonic crystal structures without asymmetries $(s=1.0)$ (results shown in Fig. 3a).

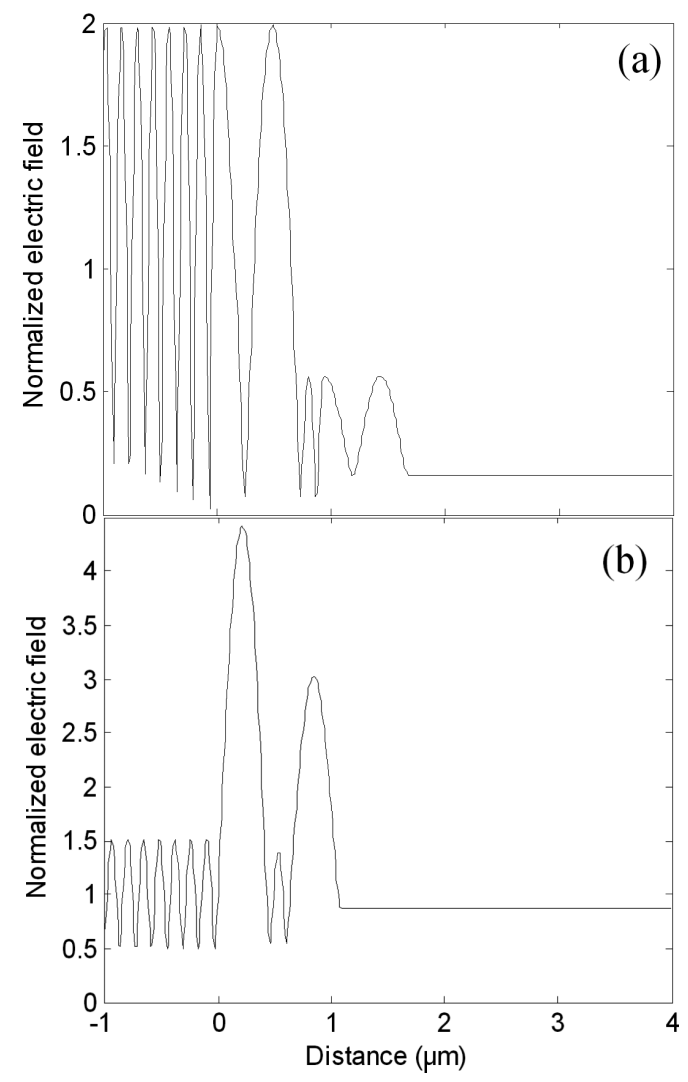

Fig. 3. Field profile for first order TE waves with normal incidence $\left(\theta_{1}=0^{\circ}\right)$ for (a) $s=1.0$, (b) $s=0.6$.

Figure 4 shows the field profiles for the first order Cantor set structure with $10^{\circ}$ angle of incidence under $s=1.0$ and $s=0.6$. Figure $4 \mathrm{a}$ and b shows the results for TE mode: the maximum normalized electric fields are 4.10 and 3.05 for $s=1.0$ and $s=0.6$, respectively. The highest normalized electric field value of approximately 4.5 is obtained for TM mode with $s=1.0$, which is shown in Fig. 4c.

Figure 5a shows the results for the reflectivity spectrum for the second order Cantor set structure with $\theta_{1}=0^{\circ}$ (normal incidence) and $s=1.0$ (solid thin black curve), 0.8 (dashed blue curve), 0.6 (solid green curve) for TE modes. The results for TE and TM modes are similar for normal incidence. There is no clear transmission window for $s=1.0$ (black curve), $s=0.8$ (blue curve) and $s=0.6$ (green curve) but two dips are observed for every values of $s$. The dips are located around $615 \mathrm{~nm}$

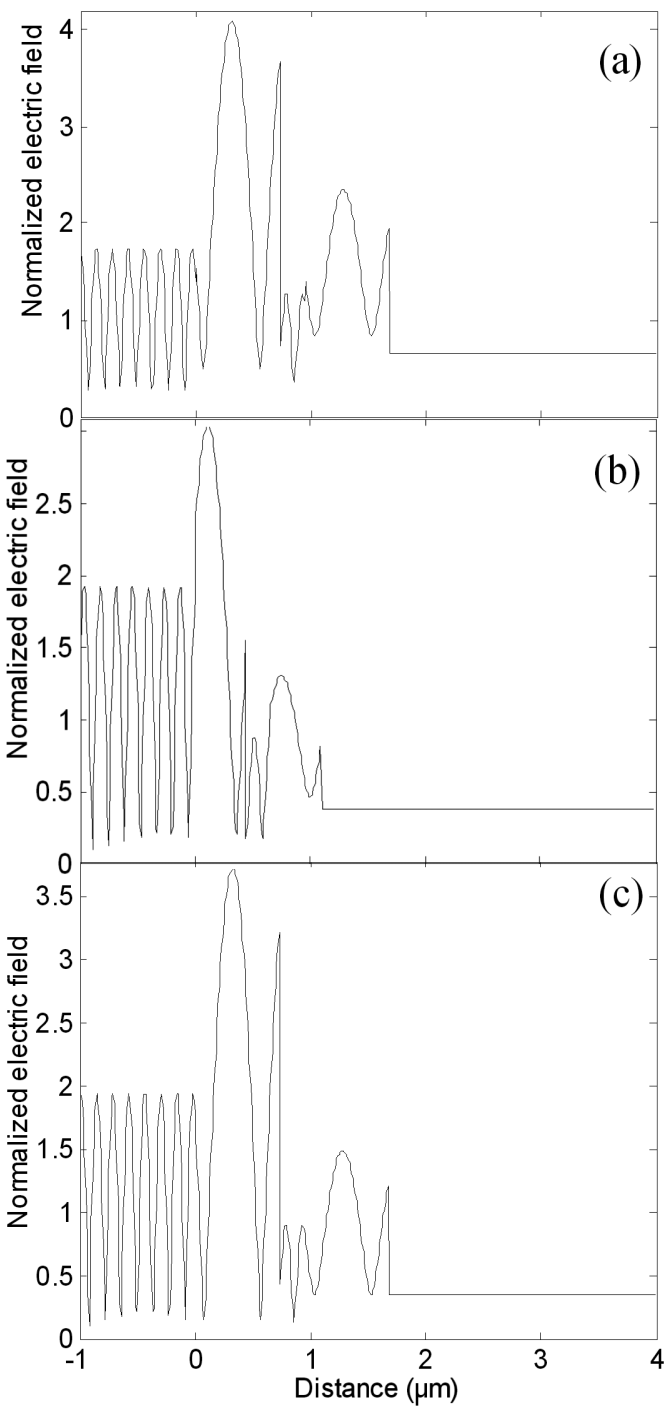

Fig. 4. Field profile for first order structure with $\theta_{1}=$ $10^{\circ}$ for (a) $\mathrm{TE}$ waves with $s=1.0$, (b) TE waves with $s=0.6$, (c) TM waves with $s=1.0$.

and $782 \mathrm{~nm}$ for $s=1.0$, while dips are at $717 \mathrm{~nm}$ and $1172 \mathrm{~nm}$ for $s=0.8$. On the other hand, the location of the two dips for asymmetry $(s=0.6)$ appears at $664 \mathrm{~nm}$ and $969 \mathrm{~nm}$. There are two bandgap (with reflectivity close to 1 ) for $s=1.0$, from approximately $611 \mathrm{~nm}$ to $771 \mathrm{~nm}$ and $772 \mathrm{~nm}$ to $1278 \mathrm{~nm}$, but only one bandgap (with reflectivity close to 1.0) exists for both $s=0.8$ (blue curve) and $s=0.6$ (green curve), are from nearly $722 \mathrm{~nm}$ to $1174 \mathrm{~nm}$ and $664 \mathrm{~nm}$ to $967 \mathrm{~nm}$, respectively.

The second order Cantor set has a layer bandgap (with reflectivity close to 1.0) for $s=1.0$ (black curve) because all layers have optical thickness $\left(d_{i} / n_{i}\right)$ multiple of a quarter wavelength: high reflectivities are expected in this case. When $s \neq 1.0$, we break and create transmission dips as can be observed in Fig. 5a.

The reflectivity spectrum for TE mode with $10^{\circ}$ incidence angle is also shown in Fig. 5b. The two clear dips 


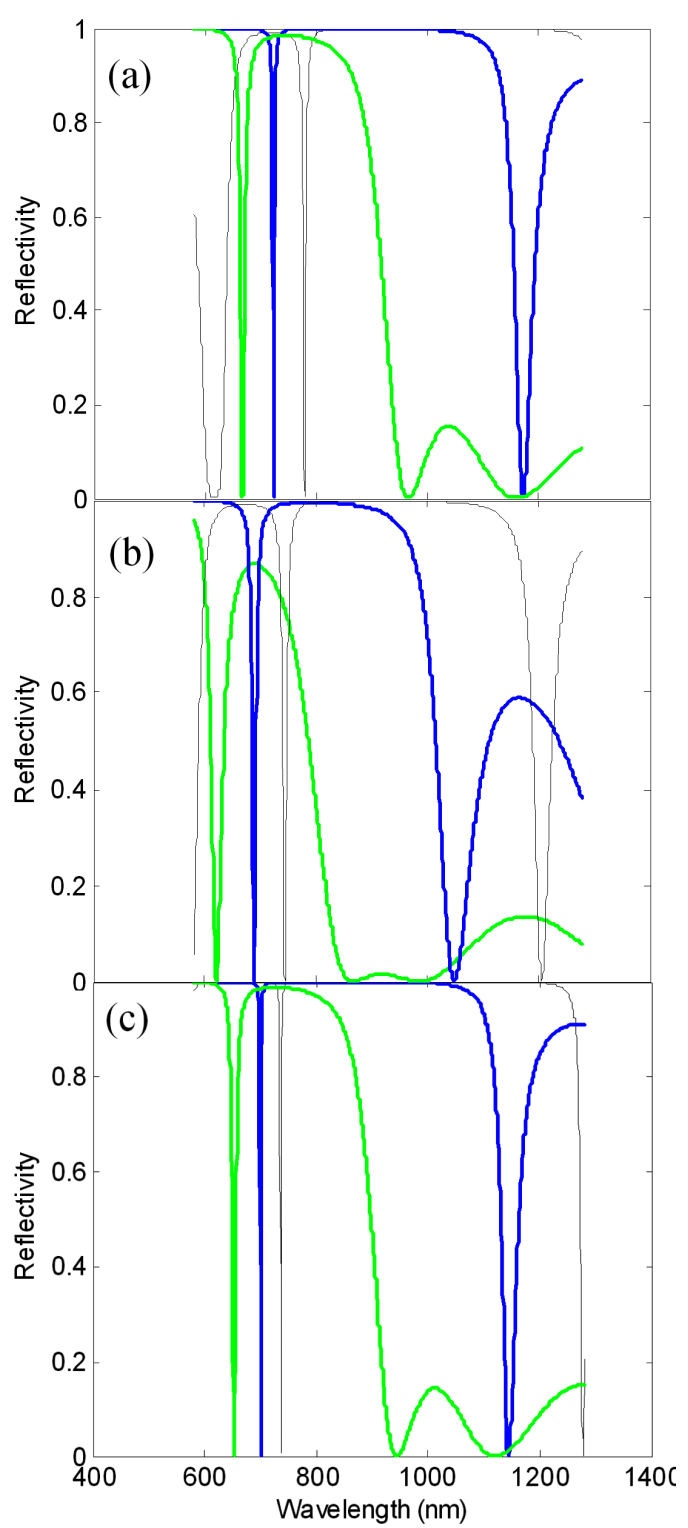

Fig. 5. Reflectivity spectrum of asymmetric Cantor set second-order GaAs-air structure for $s=1.0$ (solid thin black curve), 0.8 (dashed blue curve), 0.6 (solid thick green curve) for (a) TE mode with $\theta_{1}=0^{0}$, (b) TE mode with $\theta_{1}=10^{\circ}$, (c) TM mode with $\theta_{1}=10^{\circ}$.

are observed around at $742 \mathrm{~nm}$ and $1200 \mathrm{~nm}$ and two bandgaps (with reflectivity close to 1) occur from approximately $579 \mathrm{~nm}$ to $745 \mathrm{~nm}$ and $746 \mathrm{~nm}$ to $1204 \mathrm{~nm}$, respectively, for $s=1.0$ (black curve). In the case of asymmetry (i.e. $s=0.8$ and 0.6 ), two bandgaps (with reflectivity close to 1.0) are appeared from $689 \mathrm{~nm}$ to $1052 \mathrm{~nm}$ and $620 \mathrm{~nm}$ to $875 \mathrm{~nm}$, respectively. In addition to that there are two dips around $686 \mathrm{~nm}$ and $1055 \mathrm{~nm}$ for $s=0.8$ (dashed blue curve) and one dip at $615 \mathrm{~nm}$ for $s=0.6$ (green curve). For TM mode with $\theta_{1}=10^{\circ}$, the results are slightly different that is shown in Fig. 5(c): one dip at $736 \mathrm{~nm}$ and one bandgap (with reflectivity close to 1.0) appears between $737 \mathrm{~nm}$ to $1278 \mathrm{~nm}$ for $s=1.0$ (black curve), while two dips (at $696 \mathrm{~nm}$ and $1142 \mathrm{~nm}$ ) and one bandgap (with reflectivity close to 1.0) appears between $697 \mathrm{~nm}$ to $1147 \mathrm{~nm}$ under $s=0.8$ (dashed blue curve). In addition to that, one dip (near around $650 \mathrm{~nm}$ ) with one bandgap (with reflectivity close to 1.0) between $652 \mathrm{~nm}$ to $945 \mathrm{~nm}$ appears for $s=0.6$ (green curve).

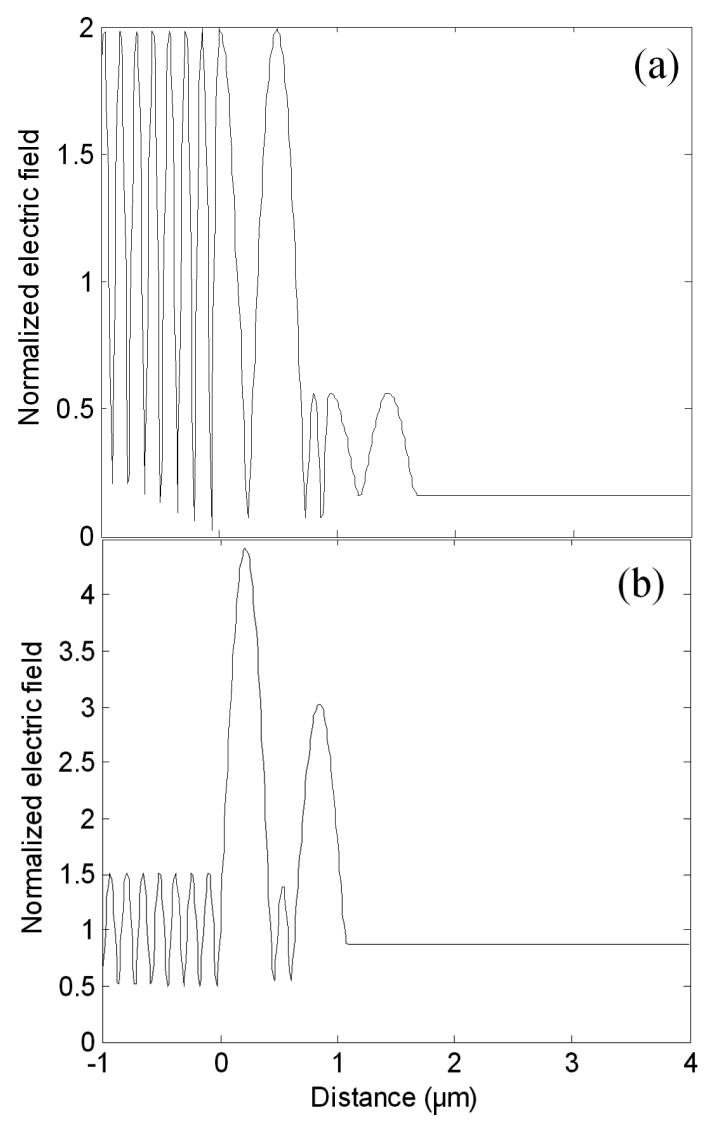

Fig. 6. Field profile of Cantor set second order structure for TE waves with normal incidence $\left(\theta_{1}=0^{\circ}\right)$ for (a) $s=1.0$, (b) $s=0.6$.

Figure $6 \mathrm{a}$ and $\mathrm{b}$ shows the field profiles as a function of distance $(\mu \mathrm{m})$ for second order Cantor set structure for TE mode with normal incidence $\left(\theta_{1}=0^{\circ}\right)$ under $s=1.0$. The maximum normalized electric field value (4.0) is observed with asymmetries $(s=0.6)$ which is double than the value obtained from structure without asymmetries. The electric field is highly localized in a very small region for $s=0.6$ and $\theta_{1}=0^{\circ}$.

Figure $7 \mathrm{a}-\mathrm{c}$ shows the field plots for second order Cantor set structure with $10^{\circ}$ angle of inclination $\left(\theta_{1}=10^{\circ}\right)$ under $s=1.0$ and $s=0.6$. Figure $7 \mathrm{a}$ and b shows the results for TE mode: the maximum normalized electric fields are 2.3 and 3.4 for $s=1.0$ and $s=0.6$, respectively. However, the electric field is increased 1.5 times after adding asymmetries $(s=0.6)$ in the original photonic crystal structures. Moreover, TM mode shows identical results as TE mode for normalized electric field profile: the value is around 2.0 as shown in Fig. 7c. 


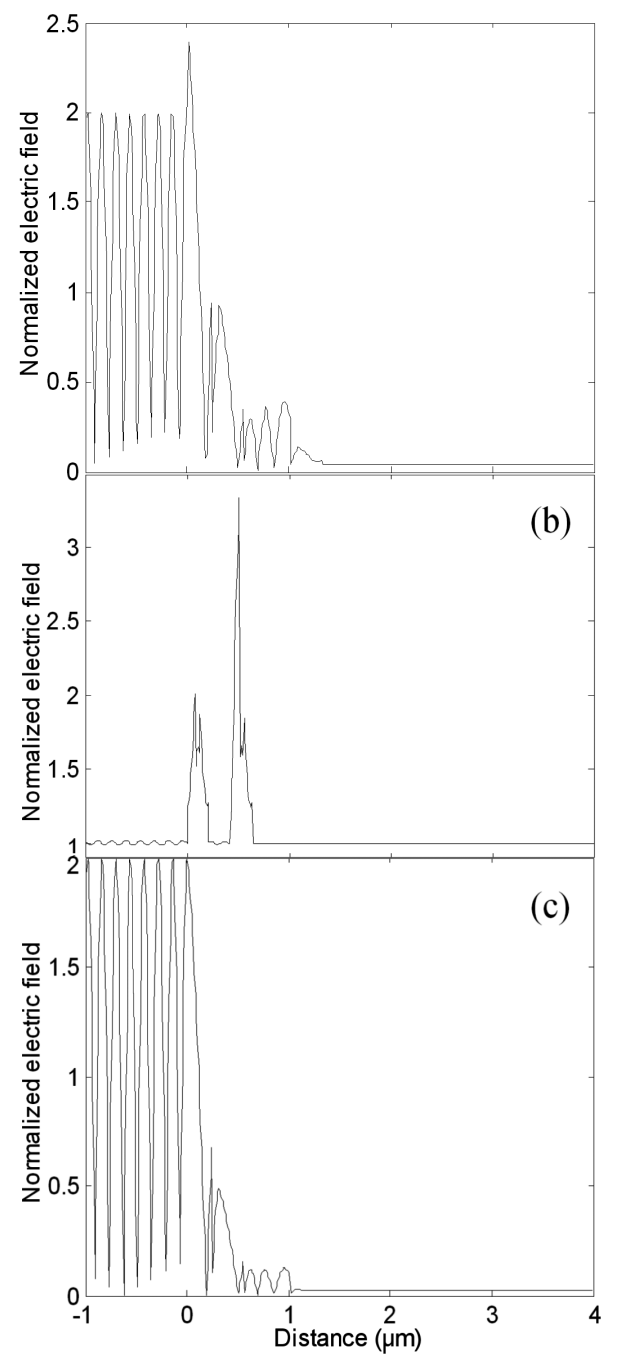

Fig. 7. Field profile for the second order Cantor set structure with $\theta_{1}=10^{\circ}$ (a) TE waves with $s=1.0$, (b) TE waves with $s=0.6$, (c) TM waves with $s=1.0$.

Now, if we replace the air layers by semiconductor layers (e.g. $\operatorname{In}_{0.2} \mathrm{Ga}_{0.8} \mathrm{As}$ ), the reflectivity will be significantly reduced to get similar values of reflectivity, a structure with many layers would be needed (for example, fiber Bragg gratings with low contrast need hundreds of periods to achieve high reflectivity).

\section{Conclusions}

In summary, asymmetric Cantor set structure based one-dimensional photonic bandgap structure is studied. It is shown that adding asymmetry in the original photonic crystal structure could tune not only the reflectivity properties of photonic crystal but also enhance the electric field. On the other hand, 2nd order Cantor set based photonic bandgap structure is not sensitive with the angular tilting of the incidence wave as the 1st order. The highest electric field is obtained for 1st order and $s=0.6$, the wave is highly localized for 2 nd order Cantor set structure. Therefore, these structures can oper- ate over larger bandwidth when compared with a classic Bragg structure with the same number of layers. It may also be more tolerant to fabrication uncertainties when compared with a Bragg structure (multiple layers that have thickness of a quarter wavelength).

\section{References}

[1] K. Falconer, Techniques in Fractal Geometry, Wiley, New York 1997.

[2] S.R. Best, IEEE Ant. Wireless Prop. Lett. 2, 197 (2003).

[3] H.T. Hattori, V.M. Schneider, O. Lisboa, J. Opt. Soc. Am. A 17, 1583 (2000).

[4] T. Okamoto, A. Fukuyama, Opt. Express 13, 8122 (2005).

[5] A.V. Lavrinenko, S.V. Zhukovsky, K.S. Sandomirski, S.V. Gaponenko, Phys. Rev. E 46, 036621 (2002).

[6] M.I. Stockman, V.M. Shalaev, M. Moskovits, R. Botet, T.F. George, Phys. Rev. B 46, 2821 (1992).

[7] G.P. Agrawal, S. Radic, IEEE Phot. Technol. Lett. 6, 995 (1994).

[8] S. Barcelos, M.N. Zervas, R.I. Laming, Opt. Fiber Technol. 2, 213 (1996).

[9] R.M. Cazo, O. Lisboa, H.T. Hattori, V.M. Schneider, C.L. Barbosa, R.C. Rabelo, J.L.S. Ferreira, Microw. Opt. Technol. Lett. 28, 4 (2001).

[10] H.T. Hattori, X. Letartre, C. Seassal, P. Rojo-Romeo, J.L. Leclercq, P. Viktorovitch, Opt. Express 11, 1799 (2003).

[11] B. Ellis, I. Fushman, D. Englund, B. Zhang, Y. Yamamoto, J. Vuckovic, Appl. Phys. Lett. 90, 151102 (2007).

[12] Q. Quan, P.B. Deotare, M. Loncar, Appl. Phys. Lett. 96, 203102 (2010).

[13] H.T. Hattori, I. McKerracher, H.H. Tan, C. Jagadish, R.M. De La Rue, IEEE J. Quantum Electron. 43, 279 (2007).

[14] A. Chutinan, S. Noda, Appl. Phys. Lett. 75, 3739 (1999).

[15] A. Mekis, J.D. Joannopoulos, J. Lightwave Technol. 19, 861 (2001).

[16] I. Udagedara, M. Premaratne, I.D. Rukhlenko, H.T. Hattori, G.P. Agrawal, Opt. Express 17, 21179 (2009).

[17] H.T. Hattori, Z. Li, D. Liu, I.D. Rukhlenko, M. Premaratne, Opt. Express 17, 20878 (2009).

[18] P. Yeh, Optical Waves in Layered Media, Wiley, New York 1988.

[19] J.D. Joannopoulos, R.D. Meade, J.N. Winn, Molding the Flow of Light, Princeton University Press, Princeton, NJ, 1995. 